Bull. Austral. Math. Soc.

VOL. 46 (1992) [373-380]

\title{
OSCILLATIONS OF CERTAIN PARTIAL DIFFERENTIAL EQUATIONS WITH DEVIATING ARGUMENTS
}

\author{
B.S. Lalli, Y.H. Yu and B.T. Cui
}

Sufficient conditions are established for the oscillation of solutions of hyperbolic equations of neutral type of the form

$$
\begin{aligned}
\frac{\partial^{2}}{\partial t^{2}}[u(x, t)+p(t) u(x, t-\tau)] & =a(t) \Delta u(x, t)+q(t) f(u(x, \sigma(t))) \\
(x, t) \in \Omega \times R_{+} & \equiv G,
\end{aligned}
$$

where $R_{+}=\{0, \infty), \Omega$ is a bounded domain in $R^{n}$ with a piecewise smooth boundary $\partial \Omega$.

Recently there has been much interest in studying the oscillatory behavior of solutions of partial differential equations with deviating arguments. We refer the reader to the papers by Georgiou and Kreith [1], Mishev and Bainov [3, 4], and Yoshida [6].

The purpose of this paper is to obtain the sufficient conditions for the oscillation of the solutions of hyperbolic equations of neutral type of the form

$$
\begin{aligned}
\frac{\partial^{2}}{\partial t^{2}}[u(x, t)+p(t) u(x, t-\tau)] & =a(t) \Delta u(x, t)+q(t) f(u(x, \sigma(t))) \\
(x, t) \in \Omega \times R_{+} & \equiv G
\end{aligned}
$$

where $R_{+}=[0, \infty), \Omega$ is a bounded domain in $R^{n}$ with a piecewise smooth bounded $\partial \Omega$.

Suppose that the following conditions (A) hold:

$\left(A_{1}\right) \quad \tau=$ const $>0, \sigma(t)$ is a continuous function in $R_{+}$such that $\lim _{t \rightarrow \infty} \sigma(t)=$ $\infty, \sigma(t) \leqslant t$

( $\left.A_{2}\right) \quad a(t)$ is a nonnegative continuous function on $R_{+}, f(u) \in C(R, R)$ are convex in $(0, \infty)$ and $u f(u)>0$ for $u \neq 0$; and

$\left(A_{3}\right) \quad q(t) \in C([0, \infty))$ and $p(t) \in C([0, \infty),[0,1])$.

Received 4th December, 1991.

Copyright Clearance Centre, Inc. Serial-fee code: 0004-9729/92 $\$ A 2.00+0.00$. 
Consider first the boundary condition

$$
\frac{\partial u}{\partial n}+\mu u=0, \quad(x, t) \in \partial \Omega \times R_{+}
$$

where $\mu$ is a continuous, nonnegative function on $\partial \Omega \times R_{+}$, and $n$ denotes the unit exterior normal vector to $\partial \Omega$.

Definition: The solution $u(u, t)$ of the problem (1), (2) is called oscillatory in $G$ if $u(x, t)$ has zero in $\Omega \times\left[t_{0}, \infty\right)$ for each $t_{0}>0$.

THEOREM 1. Let the conditions (A) hold and suppose there exists a positive constant $\alpha$ such that

$$
\frac{f(u)}{u}>\alpha \text { for } u \neq 0, \sigma^{\prime}(t) \geqslant 0 \text { for every } t \geqslant 0
$$

If

$$
\int^{\infty} q(s)[1-p(\sigma(s))] d s=\infty
$$

then every solution $u(x, t)$ of the problem (1), (2) is oscillatory.

Proof: Suppose to the contrary that there is a nonoscillatory solution $u(x, t)$ of the problem (1), (2) which has no zero in $\Omega \times\left[t_{0}, \infty\right)$. Without loss of generality we may assume that $u(x, t)>0$ in $\Omega \times\left[t_{0}, \infty\right)$. From condition $\left(A_{1}\right)$ there exists a $t_{1}>t_{0}$ such that $u(x, t)>0, u(x, \sigma(t))>0$ and $u(x, t-\tau)>0$ in $\Omega \times\left[t_{1}, \infty\right)$. We integrate (1) with respect to $x$ over the domain $\Omega$ and obtain for $t \geqslant t_{1}$

$$
\begin{aligned}
\frac{d^{2}}{d t^{2}} & {\left[\int_{\Omega} u(x, t) d x+p(t) \int_{\Omega} u(x, t-\tau) d x\right] } \\
& =a(t) \int_{\Omega} \Delta u(x, t) d x-q(t) \int_{\Omega} f(u(x, \sigma(t))) d x .
\end{aligned}
$$

Green's formula yields

$$
\int_{\mathbf{n}} \Delta u d x=\int_{\partial \Omega} \frac{\partial u}{\partial n} d s=-\int_{\theta \Omega} \mu u d s \leqslant 0 .
$$

Moreover, from $\left(A_{2}\right)$ and using Jensen's inequality it follows that

$$
\int_{\Omega} f(u(x, \sigma(t))) d x \geqslant|\Omega|\left(\frac{\int_{\Omega} u(x, \sigma(t)) d x}{|\Omega|}\right), \text { for } t \geqslant t_{1},|\Omega|=\int_{\Omega} d x .
$$

Then from (5), (6) and (7) it follows that for $t \geqslant t_{1}$

$$
\frac{d^{2}}{d t^{2}}[V(t)+p(t) V(t-\tau)]+q(t) f(V(\sigma(t))) \leqslant 0
$$


where $V(t)=\int_{\Omega} u(x, t) d x, \quad t \geqslant t_{0}$. Thus $V(t)$ is a positive solution of the inequality (8). Set $Z(t)=V(t)+p(t) V(t-\tau)$. Obviously $Z(t)>0$ for $t \geqslant t_{1}$ and

$$
Z^{\prime \prime}(t) \leqslant 0 \text { for } t \geqslant t_{1} \text {. }
$$

Hence $Z^{\prime}(t)$ is a decreasing function. We claim that $Z^{\prime}(t)>0$ for $t \geqslant t_{1}$. If $Z^{\prime}(t)<0$ for $t \geqslant t_{1}$, then there exists a $t_{2} \geqslant t_{1}$ such that $Z^{\prime}\left(t_{2}\right)<0$. Then

$$
Z(t)-Z\left(t_{2}\right) \leqslant \int_{t_{2}}^{t} Z^{\prime}\left(t_{2}\right) d s=Z^{\prime}\left(t_{2}\right)\left(t-t_{2}\right) \text { for } t \geqslant t_{2}
$$

and $\lim _{t \rightarrow \infty} Z(t)=-\infty$, which contradicts the fact that $Z(t)>0$. In view of (3), we have

or

$$
\begin{gathered}
Z^{\prime \prime}(t)+\alpha q(t) V(\sigma(t)) \leqslant 0, \text { for } t \geqslant t_{1}, \\
Z^{\prime \prime}(t)+\alpha q(t)[Z(\sigma(t))-p(\sigma(t)) V(\sigma(t)-\tau)] \leqslant 0, \quad t \geqslant t_{1} .
\end{gathered}
$$

Since $Z(t) \geqslant V(t)$ and $Z(t)$ is nondecreasing, it follows that

$$
Z^{\prime \prime}(t)+\alpha q(t)[1-p(\sigma(t))] Z(\sigma(t)) \leqslant 0, \quad t \geqslant t_{1}
$$

Let

$$
W(t)=t^{\beta} \frac{Z^{\prime}(t)}{Z(\sigma(t))}, \quad t \geqslant t_{1}, \quad \beta \geqslant 0 .
$$

We obtain for $t \geqslant t_{1}$

$$
\begin{aligned}
W^{\prime}(t) & =\beta t^{\beta-1} \frac{Z^{\prime}(t)}{Z(\sigma(t))}+t^{\beta} \frac{Z^{\prime \prime}(t) Z(\sigma(t))-Z^{\prime}(t) Z^{\prime}(\sigma(t)) \sigma^{\prime}(t)}{[Z(\sigma(t))]^{2}} \\
& =\beta t^{\beta-1} \frac{Z^{\prime}(t)}{Z(\sigma(t))}-t^{\beta} \alpha q(t)[1-p(\sigma(t))]-\frac{Z^{\prime}(t) Z^{\prime}(\sigma(t)) \sigma^{\prime}(t)}{[Z(\sigma(t))]^{2}} \\
& \leqslant \beta t^{-1} W(t)-t^{\beta} \alpha q(t)[1-p(\sigma(t))] .
\end{aligned}
$$

From this it follows that

or

$$
\begin{gathered}
\left(\frac{W(t)}{t^{\beta}}\right)^{\prime} \leqslant-\alpha q(t)[1-p(\sigma(t))] \text { for } t \geqslant t_{1}, \\
0 \leqslant \frac{W(t)}{t^{\beta}} \leqslant \frac{W\left(t_{1}\right)}{t_{1}^{\beta}}-\int_{t_{1}}^{t} \alpha q(s)[1-p(\sigma(s))] d s, \quad t \geqslant t_{1} .
\end{gathered}
$$

Thus we have

$$
\lim _{t \rightarrow \infty} \frac{W(t)}{t^{\beta}}=-\infty
$$

which leads to a contradiction.

If $u(x, t) \leqslant 0$ for $(x, t) \in \Omega \times\left[t_{0}, \infty\right)$, then the proof follows from the fact that $-u(x, t)$ is a positive solution of the problem (1), (2). This completes the proof of the theorem. 
REMARK. If $u(x, t) \equiv u(t), f(u) \equiv u$ and $\sigma=t-\sigma$, then Theorem 1 and the Theorem in [2] are the same.

We consider now the boundary condition

$$
u=0, \quad \text { on } \partial \Omega \times[0, \infty),
$$

and we consider the following Dirichlet problem in the domain $\Omega$

$$
\begin{aligned}
\Delta v+\alpha v & =0, \quad \text { in } \Omega \\
\left.v\right|_{\partial \Omega} & =0
\end{aligned}
$$

where $\alpha$ is a constant. It is well-known that the smallest eigenvalue $\alpha_{0}$ of the problem (12) is positive and the corresponding eigenfunction $\psi(x) \geqslant 0$ for $x \in \Omega$.

With a solution $u(x, t)$ of the problem (1), (11) we associate the function

$$
H(t)=\frac{\int_{\Omega} u(x, t) \psi(x) d x}{\int_{\Omega} \psi(x) d x}, \quad t \geqslant 0
$$

THEOREM 2. If all conditions of Theorem 1 hold, then every solution of the problem (1), (11) is oscillatory in $G$.

Proof: Let $u(x, t)$ be a positive solution of the problem (1), (11) in $\Omega \times\left[t_{0}, \infty\right)$ for some $t_{0}$. By condition $\left(A_{1}\right)$ there exists a $t_{1} \geqslant t_{0}$ such that $u(x, \sigma(t)) \geqslant 0$ and $u(x, t-\tau)>0$ in $\Omega \times\left[t_{1}, \infty\right)$. Multiply both sides of equation (1) by the eigenfunction $\psi(x) \geqslant 0$, and integrate with respect to $x$ over the domain $\Omega$; then we have

$$
\begin{aligned}
& \frac{d^{2}}{d t^{2}}\left[\int_{\Omega} u(x, t) \psi(x) d x+p(t) \int_{\Omega} u(x, t-\tau) \psi(x) d x\right] \\
& \quad=a(t) \int_{\Omega} \Delta u \psi(x) d x-q(t) \int_{\Omega} f(u(x, \sigma(t))) \psi(x) d x, \quad t>t_{1}
\end{aligned}
$$

From the divergence theorem it follows that

$$
\int_{\Omega} \Delta u \psi(x) d x=-\alpha_{0} \int_{\Omega} u \psi(x) d x, \quad t \geqslant t_{1},
$$

where $\alpha_{0}$ is the smallest eigenvalue of the problem (12).

Using condition $\left(A_{2}\right)$ and Jensen's inequality it follows that

$$
\begin{aligned}
\int_{\Omega} f & (u(x, \sigma(t))) \psi(x) d x \\
& \geqslant \int_{\Omega} \psi(x) d x f\left(\frac{1}{\int_{\Omega} \psi(x) d x} \int_{\Omega} u(x, \sigma(t)) \psi(x) d x\right), \quad t \geqslant t_{1} .
\end{aligned}
$$


Using (13), (15) and (16), we obtain

$$
\frac{d^{2}}{d t^{2}}[H(t)+p(t) H(t-\tau)] \leqslant-\alpha_{0} a(t) H(t)-q(t) f(H(\sigma(t))), \quad t \geqslant t_{1} .
$$

Since, for $t \geqslant t_{1} H(t) \geqslant 0$ and $H(\sigma(t)) \geqslant 0$, then by (17)

$$
\frac{d^{2}}{d t^{2}}[H(t)+p(t) H(t-\tau)]+q(t) f(H(\sigma(t))) \leqslant 0, \quad t \geqslant t_{1} .
$$

The remainder of the proof is similar to that of Theorem 1; we omit it.

We need the following lemma [5].

LEMma. Consider the differential inequality

$$
x^{\prime}(t)+b(t) x(g(t)) \leqslant 0, \quad t \geqslant t_{0},
$$

where $b(t) \in C(R,[0, \infty)), g(t) \in C(R, R), g(t) \leqslant t$ and $g(t)$ is a nondecreasing function with $\lim _{t \rightarrow \infty} g(t)=\infty$. If

$$
\liminf _{t \rightarrow \infty} \int_{g(t)}^{t} b(s) d s \geqslant \frac{1}{e},
$$

then the inequality (18) has no ultimately positive solutions.

Theorem 3. Let conditions (A) and (3) hold. If

$$
\liminf _{t \rightarrow \infty} \int_{\sigma(t)}^{t} Q(s) d s>\frac{1}{e}
$$

where $Q(t)=\alpha_{0} \varepsilon_{0} q(t) \sigma(t)[1-p(\sigma(t))] \exp \left[\int_{T}^{t} \alpha_{0} \varepsilon_{0} a(s) s[1-p(s)] d s\right]$,

for all large $T, \varepsilon_{0} \in(0,1)$ is a constant, then every solution $u(x, t)$ of the problem (1), (11) is oscillatory in $G$.

Proof: Let $u(x, t)$ be a positive solution of the problem (1)-(11). As in the proof of Theorem 2 we get (17). From (17) and (3) it follows that

$$
\frac{d^{2}}{d t^{2}}[H(t)+p(t) H(t-\tau)]+\alpha_{0} a(t) H(t)+\alpha q(t) H(\sigma(t)) \leqslant 0, \quad t \geqslant t_{1} .
$$

Moreover,

$$
\begin{gathered}
Z^{\prime \prime}(t)+\alpha_{0} a(t)[Z(t)-p(t) H(t-\tau)]+\alpha q(t)[Z(\sigma(t))-p(\sigma(t)) H(\sigma(t)-\tau)] \leqslant 0 \\
t \geqslant t_{1} .
\end{gathered}
$$


Since $Z(t) \geqslant H(t)$ and $Z(t)$ is nondecreasing, we have

$$
Z^{\prime \prime}(t)+\alpha_{0} a(t)[1-p(t)] Z(t)+\alpha q(t)[1-p(\sigma(t))] Z(\sigma(t)) \leqslant 0, \quad t \geqslant t_{1} .
$$

Obviously, $Z(t) \geqslant 0, Z^{\prime \prime}(t) \leqslant 0$ and $Z^{\prime} \geqslant 0$ for $t \geqslant t_{1}$ (as in the proof of Theorem 1 ). By Lemma 1 in [5] there exists a $T \geqslant t_{1}$ such that

$$
Z(t) \geqslant \varepsilon_{0} t Z^{\prime}(t) \text { and } Z(\sigma(t)) \geqslant \varepsilon \sigma(t) Z^{\prime}(\sigma(t)) \text { for } t \geqslant T .
$$

Hence, from (20) we have

$$
Z^{\prime \prime}(t)+\alpha_{0} \varepsilon_{0} T a(t)[1-p(t)] Z^{\prime}(t)+\alpha \varepsilon_{0} q(t) \sigma(t)[1-p(\sigma(t))] Z^{\prime}(\sigma(t)) \leqslant 0, \quad t \geqslant T .
$$

Set

$$
Y(t)=Z^{\prime}(t) \exp \left[\int_{t_{2}}^{t} \alpha_{0} \varepsilon_{0} s a(s)[1-p(s)]\right] d s, \quad t \geqslant T
$$

and using (21) we obtain

$$
Y^{\prime}(t)+Q(t) Y(\sigma(t)) \leqslant 0, \quad t \geqslant T
$$

where $Q(t)=\alpha_{0} \varepsilon_{0} q(t) \sigma(t)[1-p(\sigma(t))] \exp \left[\int_{T}^{t} \alpha_{0} \varepsilon_{0} a(s) s[1-p(s)]\right] d s$.

Thus (22) has a positive solution $Y(t)$, which contradicts the conclusion of the lemma.

THEOREM 4. Let conditions (A) and (3) hold. If

$$
\underset{t \rightarrow \infty}{\limsup } \int_{\sigma(t)}^{t} \alpha_{0} q(t) \sigma(t)[1-p(\sigma(t))] d t \geqslant 1
$$

then every solution $u(x, t)$ of the problem (1), (11) is oscillatory.

Proof: As in the proof of Theorem 3, we obtain (21). Thus

$$
Y^{\prime}(t)+\alpha_{0} \varepsilon_{0} q(t) \sigma(t)[1-p(\sigma(t))] Y(\sigma(t)) \leqslant 0, \quad t \geqslant T
$$

where $Y(t)=Z^{\prime}(t), \varepsilon_{0} \in(0,1)$, such that

$$
\limsup _{t \rightarrow \infty} \int_{\sigma(t)}^{t} \alpha_{0} \varepsilon_{0} q(s) \sigma(s)[1-p(\sigma(s))] d s \geqslant 1
$$

Integrating (23) from $\sigma(t)$ to $t$, we have

$$
Y(t)-Y(\sigma(t))+\alpha_{0} \varepsilon_{0} \int_{\sigma(t)}^{t} q(s) \sigma(s)[1-p(\sigma(s))] Y(\sigma(s)) d s \leqslant 0, \quad t \geqslant T
$$


Since $Y(t)$ is decreasing and $\sigma^{\prime}(t) \geqslant 0$, it follows that

$$
\begin{array}{cc}
Y(t)-Y(\sigma(t))+\alpha_{0} \varepsilon_{0} Y(\sigma(t)) \int_{\sigma(t)}^{t} q(s) \sigma(s)[1-p(\sigma(s))] d s \leqslant 0, \quad t \geqslant T, \\
\text { or } \quad \alpha_{0} \varepsilon_{0} \int_{\sigma(t)}^{t} q(s) \sigma(s)[1-p(\sigma(s))] d s \leqslant 1-\frac{Y(t)}{Y(\sigma(t))} \leqslant 1, \quad t \geqslant T . \\
\text { Hence } \\
\quad \limsup _{t \rightarrow \infty} \int \alpha_{0} \varepsilon_{0} q(s) \sigma(s)[1-p(\sigma(s))] d s \leqslant 1,
\end{array}
$$

which contradicts (24). Thus the proof of Theorem 4 is complete.

EXAmple 1. Consider the equation

$$
\begin{aligned}
\frac{\partial^{2}}{\partial t^{2}} & {\left[u(x, t)+\frac{1}{2} u\left(x, t-\frac{\pi}{2}\right)\right] } \\
& =u_{x x}(x, t)-\frac{1}{2} u\left(x, t-\frac{\pi}{2}\right), \quad(x, t) \in(0, \pi) \times[0, \infty),
\end{aligned}
$$

with a boundary condition

$$
u_{x}(0, t)=u_{x}(\pi, t)=0, \quad t \geqslant 0 .
$$

It is easy to check the functions: $f(u)=u, q(t)=1 / 2=p(t), a(t)=1, \tau=\pi / 2$ and $\alpha=1$. Moreover, $u \equiv 0$ on $\partial \Omega \times[0, \infty)$ where $\Omega=(0, \pi)$, and

$$
\int^{\infty} q(s)[1-p(\sigma(s))] d s=\int^{\infty} \frac{1}{2}\left[1-\frac{1}{2}\right] d s=\infty .
$$

Hence the conditions of Theorem 1 are satisfied. Thus all solutions of the problem (25), (26) oscillate in $G=(0, \pi) \times[0, \infty)$. For instance the function $u(x, t)=\sin t \cos x$ is such a solution.

EXAMPLE 2. Consider the equation

$$
\begin{aligned}
\frac{\partial^{2}}{\partial t^{2}}\left[u(x, t)+e^{-\pi} u(x, t-\pi)\right]= & u_{x x}(x, t)-e^{-\pi} u(x, t-\pi), \\
& (x, t) \in(0, \pi) \times[0, \infty),
\end{aligned}
$$

with a boundary condition

$$
u(0, t)=u(\pi, t)=0, \quad t \geqslant 0 .
$$

It is easily verified that the functions

$$
p(t)=e^{-\pi}, q(t)=e^{-\pi}, a(t)=1, \sigma(t)=t-\pi \quad \text { and } \quad f(u)=u
$$

satisfy the conditions of Theorem 4. Hence all solutions of the problem (27), (28) oscillate in $G=(0, \pi) \times[0, \infty)$. For instance, the function $u(x, t)=\sin t \sin x$ is $\operatorname{such}$ a solution. 


\section{REFERENCES}

[1] D. Georgiou and K. Kreith, 'Functional characteristic initial value problems', J. Math. Anal. Appl. 107 (1985), 414-424.

[2] M.K. Grammatikopoulos, G. Ladas and A. Meimaridou, 'Oscillations of Second Order neutral delay differential equations', Rad. Mat. 1 (1985), 267-274.

[3] D.P. Mishev and D.D. Bainov, 'Oscillation properties of the solutions of a class of hyperbolic equations of neutral type', Funkcial. Ekvac. 29 (1986), 213-218.

[4] D.P. Mishev and D.D. Bainov, 'Oscillation of the solutions of parabolic differential equations of neutral type', Appl. Math. Comput. 28 (1988), 97-111.

[5] Wei Junjie, 'Oscillation of second order delay differential equations', Ann. Differential Eqations 4 (1988), 473-478.

[6] N. Yoshid, 'Forced oscillations of solutions of parabolic equations', Bull. Austral. Math. Soc. 36 (1987), 289-294.

Department of Mathematics

University of Saskatchewan

Saskatoon, Saskatchewan S7N 0W0

Canada

Bin Zhou Normal College

Shandong

China 256604
Institute of Applied Mathematics

Academia Sinica

Beijing

China 100080 